\title{
Unitary Dynamics of Strongly Interacting Bose Gases with the Time-Dependent Variational Monte Carlo Method in Continuous Space
}

\author{
Giuseppe Carleo \\ Institute for Theoretical Physics, ETH Zurich, Wolfgang-Pauli-Strasse 27, 8093 Zurich, Switzerland \\ Lorenzo Cevolani \\ Laboratoire Charles Fabry, Institut d'Optique, CNRS, Univ Paris Sud 11, \\ 2 avenue Augustin Fresnel, F-91127 Palaiseau cedex, France \\ Laurent Sanchez-Palencia \\ Laboratoire Charles Fabry, Institut d'Optique, CNRS, Univ Paris Sud 11, 2 avenue Augustin Fresnel, \\ F-91127 Palaiseau cedex, France \\ and Centre de Physique Théorique, Ecole Polytechnique, CNRS, Univ Paris-Saclay, \\ F-91128 Palaiseau, France \\ Markus Holzmann \\ LPMMC, UMR 5493 of CNRS, Université Grenoble Alpes, F-38042 Grenoble, France \\ and Institut Laue-Langevin, BP 156, F-38042 Grenoble Cedex 9, France
}

(Received 19 December 2016; revised manuscript received 6 April 2017; published 8 August 2017)

\begin{abstract}
We introduce the time-dependent variational Monte Carlo method for continuous-space Bose gases. Our approach is based on the systematic expansion of the many-body wave function in terms of multibody correlations and is essentially exact up to adaptive truncation. The method is benchmarked by comparison to an exact Bethe ansatz or existing numerical results for the integrable Lieb-Liniger model. We first show that the many-body wave function achieves high precision for ground-state properties, including energy and first-order as well as second-order correlation functions. Then, we study the out-of-equilibrium, unitary dynamics induced by a quantum quench in the interaction strength. Our time-dependent variational Monte Carlo results are benchmarked by comparison to exact Bethe ansatz results available for a small number of particles, and are also compared to quench action results available for noninteracting initial states. Moreover, our approach allows us to study large particle numbers and general quench protocols, previously inaccessible beyond the mean-field level. Our results suggest that it is possible to find correlated initial states for which the long-term dynamics of local density fluctuations is close to the predictions of a simple Boltzmann ensemble.
\end{abstract}

DOI: 10.1103/PhysRevX.7.031026

\author{
Subject Areas: Atomic and Molecular Physics, \\ Computational Physics, \\ Quantum Physics
}

\section{INTRODUCTION}

The study of equilibration and thermalization properties of complex many-body systems is of fundamental interest for many areas of physics and natural sciences [1]. For systems governed by classical physics, an exact solution of Newton's equations of motion is often numerically feasible, using, for instance, molecular-dynamics simulations. For quantum systems, the mathematical structure of the timedependent Schrödinger equation is instead fundamentally

Published by the American Physical Society under the terms of the Creative Commons Attribution 4.0 International license. Further distribution of this work must maintain attribution to the author(s) and the published article's title, journal citation, and DOI. more involved. Quantum Monte Carlo algorithms, the de facto tool for simulating quantum many-body systems at thermal equilibrium [2-4], cannot be directly used to study time-dependent unitary dynamics. Out-of-equilibrium properties are then often treated on the basis of approximations drastically simplifying the microscopic physics. Irreversibility is either enforced with an explicit breaking of unitarity, e.g., within the quantum Boltzmann approach, or the dynamics is reduced to mean-field description using time-dependent Hartree-Fock and Gross-Pitaevskii approaches. Although these approaches may qualitatively describe thermalization $[5,6]$, their range of validity cannot be assessed because genuine quantum correlations and entanglement are ignored.

For specific systems, exact dynamical results can be derived. This is the case for integrable 1D models, for which Bethe ansatz (BA) solutions exist [7]. However, also 
in this case many open questions still persist. For example, the exact evaluation of correlation functions for out-ofequilibrium dynamics is at present an unsolved problem. As a result, despite important theoretical and experimental progress [8-15], a complete picture of thermalization (or its absence), e.g., based on general quench protocols [16], is still missing.

Numerical methods for strongly interacting systems face important challenges as well. Numerical renormalization group and density-matrix renormalization group (DMRG) approaches provide an essentially exact description of arbitrary 1D lattice systems in and out of equilibrium [17-20], but they have less predictive power when applied to continuous-space systems. On one hand, multiscale extensions of the DMRG optimization scheme to the limit of continuous-space lattices [21] are, to date, limited to relatively small system sizes [22]. On the other hand, efficient ground-state optimization schemes for continuous quantum field matrix product states (cMPS) [23] have been introduced only very recently [24], and applications to quantum dynamics are still to be realized. A further formidable challenge is the efficient extension of these approaches to higher dimensions, which is a fundamentally hard problem.

Another class of methods for strongly interacting systems is based on variational Monte Carlo (VMC) methods, combining highly entangled variational states with robust stochastic optimization schemes [25]. Such approaches have been successfully applied to the description of continuous quantum systems, in any dimension and not only in 1D [26,27]. More recently, out-of-equilibrium dynamics has become accessible with the extension of these methods to real-time unitary dynamics, within the time-dependent variational Monte Carlo (tVMC) method [28,29]. So far, the tVMC approach has been developed for lattice systems with bosonic [28,29], spin [30-32], and fermionic [33] statistics, yielding a description of dynamical properties with an accuracy often comparable with MPS-based approaches.

In this paper, we extend the tVMC approach to access dynamical properties of interacting quantum systems in continuous space. Our approach is based on a systematic expansion of the wave function in terms of fewbody Jastrow correlation functions. Using the 1D LiebLiniger model as a test case, we first show that the inclusion of high-order correlations allows us to systematically approach the exact BA ground-state energy. Our results improve by orders of magnitude on previously published VMC and cMPS results, and are in line with the latest stateof-the-art developments in the field. We further compute single-body and pair correlation functions, hardly accessible by current BA methods. We then calculate the time evolution of the contact pair correlation function following a quench in the interaction strength. For the noninteracting initial state, we benchmark our results to exact BA calculations available for a small number of bosons and further compare to the quench action approach for large systems approaching the thermodynamic limit. Finally, we apply our method to the study of general quenches from arbitrary initial states, for which no exact results in the thermodynamic limit are currently available.

\section{METHOD}

\section{A. Expansion of the many-body wave function}

Consider a nonrelativistic quantum system of $N$ identical bosons in $d$ dimensions, and governed by the firstquantization Hamiltonian

$$
\mathcal{H}=-\frac{1}{2} \sum_{i=1}^{N} \nabla_{i}^{2}+\sum_{i=1}^{N} v_{1}\left(\vec{x}_{i}\right)+\frac{1}{2} \sum_{i \neq j} v_{2}\left(\vec{x}_{i}, \vec{x}_{j}\right),
$$

where $v_{1}(\vec{x})$ and $v_{2}(\vec{x}, \vec{y})$ are, respectively, a one-body external potential and a pairwise interparticle interaction [34]. Without loss of generality, a time-dependent $N$-body state can be written as $\Phi(\mathbf{X}, t)=\exp [U(\mathbf{X} ; t)]$, where $\mathbf{X}=\vec{x}_{1}, \vec{x}_{2}, \ldots, \vec{x}_{N}$ is the ensemble of particle positions and $U$ is a complex-valued function of the $N$-particle coordinates, $\mathbb{R}^{N \times d} \rightarrow \mathbb{C}$. Since the Hamiltonian Eq. (1) contains only two-body interactions, it is expected that an expansion of $U$ in terms of few-body Jastrow functions containing at most $m$-body terms rapidly converges towards the exact solution. Truncating this expansion up to a certain order, $M \leq N$, leads to the Bijl-Dingle-JastrowFeenberg expansion [35-38],

$$
\begin{aligned}
U^{(M)}(\mathbf{X} ; t)= & \sum_{i=1} u_{1}\left(\vec{x}_{i} ; t\right)+\frac{1}{2 !} \sum_{i \neq j} u_{2}\left(\vec{x}_{i}, \vec{x}_{j} ; t\right) \\
& +\cdots \frac{1}{M !} \sum_{i_{1} \neq i_{2} \neq \ldots i_{M}} u_{M}\left(\vec{x}_{i_{1}}, \vec{x}_{i_{2}}, \ldots, \vec{x}_{i_{M}} ; t\right),
\end{aligned}
$$

where $u_{m}(\mathbf{r} ; t)$ are functions of $m$ particle coordinates, $\mathbf{r}=\vec{x}_{i_{1}}, \vec{x}_{i_{2}}, \ldots, \vec{x}_{i_{m}}$, and of the time $t$. A global constraint on the function $U(\mathbf{X}, t)$ is given by particle statistics. In the bosonic case, we demand that $U\left(\vec{x}_{1}, \vec{x}_{2}, \ldots, \vec{x}_{N}\right)=$ $U\left(\vec{x}_{\sigma(1)}, \vec{x}_{\sigma(2)}, \ldots, \vec{x}_{\sigma(N)}\right)$, for all particle permutations $\sigma$. In general, the functions $u_{m}(\mathbf{r} ; t)$ can have an arbitrarily complex dependence on the $m$ particle coordinates, which can prove problematic for practical applications. Nonetheless, a simplified functional dependence can often be imposed, resulting from the two-body character of the interactions in the original Hamiltonian. For $m \geq 3$, $u_{m}(\mathbf{r} ; t)$ can be conveniently factorized in terms of general two-particle vector and tensor functions following Ref. [26]. Details of this approach and the present implementation are presented in Appendixes A and F.

An appealing property of the many-body expansion Eq. (2) is that it is able to describe intrinsically nonlocal correlations in space. For instance, the two-body $u_{2}\left(\vec{x}_{i}, \vec{x}_{j} ; t\right)$, as well as any $m$-body function $u_{m}(\mathbf{r} ; t)$, 
can be long range in the particle separation $\left|\vec{x}_{i}-\vec{x}_{j}\right|$. This nonlocal spatial structure allows for a correct description of gapless phases, where a two-body expansion may already capture all the universal features, in the sense of the renormalization group approach [39]. This is in contrast with the MPS decomposition of the wave function, which is intrinsically local in space.

\section{B. Time-dependent variational Monte Carlo method}

The time evolution of the variational state Eq. (2) is entirely determined by the time dependence of the Jastrow functions $u_{m}(\mathbf{r} ; t)$. In order to establish optimal equations of motion for the variational parameters, we note that the functional derivative of $U(\mathbf{X} ; t)$ with respect to the variational, complex-valued, Jastrow functions $u_{m}(\mathbf{r} ; t)$,

$$
\frac{\delta U(\mathbf{X} ; t)}{\delta u_{m}(\mathbf{r} ; t)} \equiv \rho_{m}(\mathbf{r})
$$

yields the $m$-body density operators

$$
\rho_{m}(\mathbf{r})=\frac{1}{m !} \sum_{i_{1} \neq i_{2} \neq \cdots i_{m}} \prod_{j} \delta\left(x_{i_{j}}-r_{j}\right) .
$$

The expectation values of the operators $\rho_{m}$ over the state $|\Phi(t)\rangle$ give the instantaneous $m$-body correlations. For instance, $\left\langle\rho_{2}\left(r_{1}, r_{2}\right)\right\rangle_{t}=\sum_{i<j}\left\langle\delta\left(x_{i}-r_{1}\right) \delta\left(x_{j}-r_{2}\right)\right\rangle_{t}$, where $\langle\cdots\rangle_{t}=\langle\Phi(t)|\ldots| \Phi(t)\rangle /\langle\Phi(t) \mid \Phi(t)\rangle$, is proportional to the two-point density-density correlation function.

We can then express the time derivative of the truncated variational state $U^{(M)}(\mathbf{X} ; t)$ using the functional derivatives, Eq. (3), as a sum of the few-body density operators up to the truncation $M$; i.e.,

$$
\partial_{t} U^{(M)}(\mathbf{X}, t)=\sum_{m=1}^{M} \int d \mathbf{r} \rho_{m}(\mathbf{r}) \partial_{t} u_{m}(\mathbf{r} ; t) .
$$

The exact wave function satisfies the Schrödinger equation $i \partial_{t} U(\mathbf{X} ; t)=E_{\mathrm{loc}}(\mathbf{X}, t)$, where $E_{\mathrm{loc}}(\mathbf{X} ; t)=$ $(\langle\mathbf{X}|\mathcal{H}| \Phi(t)\rangle /\langle\mathbf{X} \mid \Phi(t)\rangle)$ is the so-called local energy. The optimal time evolution of the truncated Bijl-DingleJastrow-Feenberg expansion Eq. (2) can be derived imposing the Dirac-Frenkel time-dependent variational principle [40,41]. In geometrical terms, this amounts to minimizing the Hilbert-space norm of the residuals $R^{(M)}(\mathbf{X} ; t) \equiv\left\|i \partial_{t} U^{(M)}(\mathbf{X} ; t)-E_{\mathrm{loc}}^{(M)}(\mathbf{X} ; t)\right\|$, thus yielding a variational many-body state as close as possible to the exact one [42]. The minimization can be performed explicitly and yields a closed set of integro-differential equation for the Jastrow functions $u_{m}(\mathbf{r} ; t)$ :

$$
\sum_{p=1}^{M} \int d \mathbf{r}^{\prime} \frac{\delta\left\langle\rho_{m}(\mathbf{r})\right\rangle_{t}}{\delta u_{p}\left(\mathbf{r}^{\prime} ; t\right)} \partial_{t} u_{p}\left(\mathbf{r}^{\prime} ; t\right)=-i \frac{\delta\langle\mathcal{H}\rangle_{t}}{\delta u_{m}(\mathbf{r} ; t)}
$$

In practice, these equations are numerically solved for the time derivatives $\partial_{t} u_{p}\left(\mathbf{r}^{\prime} ; t\right)$ at each time step $t$. The expectation values taken over the time-dependent state $\langle\cdot\rangle_{t}$, which enter Eq. (6), are found via a stochastic sampling of the probability distribution $\Pi(\mathbf{X}, t)=|\Phi(\mathbf{X}, t)|^{2}$. This is efficiently achieved by means of the Metropolis-Hastings algorithm, as per conventional Monte Carlo schemes (see Appendix B for details). It then yields the full time evolution of the truncated Bijl-Dingle-Jastrow-Feenberg state Eq. (2) after time integration.

The tVMC approach as formulated here provides, in principle, an exact description of the real-time dynamics of the $N$-body system. The essential approximation lies, however, in the truncation of the Bijl-Dingle-JastrowFeenberg expansion to the $M$ most relevant terms. In practical applications, the $M=2$ or $M=3$ truncation is often sufficient. Systematic improvement beyond $M=3$ is possible [26], but may require substantial computational effort.

\section{LIEB-LINIGER MODEL}

As a first application of the continuous-space tVMC approach, we consider the Lieb-Liniger model [43]. On one hand, some exact results and numerical data are available, allowing us to benchmark the Jastrow expansion and the tVMC approach. On the other hand, several aspects of the out-of-equilibrium dynamics of this model are unknown, which we compute here for the first time using the tVMC approach.

The Lieb-Liniger model describes $N$ interacting bosons in one dimension with contact interactions. It corresponds to the Hamiltonian Eq. (1) with $v_{1}(x)=0$ and $v_{2}(x, y)=$ $g \delta(x-y)$, where $g$ is the coupling constant. Here, we consider periodic boundary conditions over a ring of length $L$ and the particle density $n=N / L$. The density dependence is as usual expressed in terms of the dimensionless parameter $\gamma=m g / \hbar^{2} n$. The Lieb-Liniger model is the prototypal model of continuous one-dimensional strongly correlated gas exactly solvable by the Bethe ansatz [43]. This model is experimentally realized in ultracold atomic gases strongly confined in one-dimensional optical traps, and several studies on out-of-equilibrium physics have already been realized [14,44-48].

As a result of translation invariance, we have $u_{1}(x ; t)=$ const, and the first nontrivial term in the many-body expansion Eq. (2) is the two-body, translation invariant, function $u_{2}(|x-y| ; t)$. To compute the functional derivatives of the many-body wave function, we proceed with a projection of the continuous Jastrow fields $u_{m}(\mathbf{r} ; t)$ onto a finite-basis set. Here, we find it convenient to represent both the field Hamiltonian Eq. (1) and the Jastrow functions on a uniform mesh of spacing $a$, leading to $n_{\mathrm{var}}=$ $[L /(2 a)]$ variational parameters for the two-body Jastrow term. In the following, our results are extrapolated to the continuous limit, corresponding to $a \rightarrow 0$. The finite-basis 
projection as well as the numerical time integration of Eq. (6) are detailed in Appendix $\mathrm{C}$ and benchmarked against exact diagonalization results for $N=3$ in Appendix E.

\section{A. Ground-state properties}

To assess the quality of truncated Bijl-Dingle-JastrowFeenberg expansions for the Lieb-Liniger model, we start our analysis considering ground-state properties. An exact solution can be found from the Bethe ansatz and gives access to exact ground-state energies and local properties [43]. Other nonlocal properties are substantially more difficult to extract from the BA solution, and unbiased results for ground-state correlation functions have not been reported so far. In order to determine the best possible variational description of the ground state within our manybody expansion, different strategies are possible. A first possibility is to consider the imaginary-time evolution $|\Psi(\tau)\rangle=e^{-\tau \mathcal{H}}\left|\Psi_{0}\right\rangle$, which systematically converges to the exact ground state in the limit $\tau \gg \Delta_{1}$, where $\Delta_{1}=$ $E_{1}-E_{0}$ is the gap with the first excited state on a finite system and provided that the trial state $\Psi_{0}$ is nonorthogonal to the exact ground state. Imaginary-time evolution in the variational subspace can be implemented considering the formal substitution $t \rightarrow-i \tau$ in the tVMC equations [Eq. (6)]. The resulting equations are equivalent to the stochastic reconfiguration approach [49]. However, direct minimization of the variational energy can be significantly more efficient, in particular, for systems becoming gapless in the thermodynamic limit, where $\Delta_{1} \sim \operatorname{poly}(1 / N)$. Given the gapless nature of the Lieb-Liniger model, we find it computationally more efficient to adopt a Newton method to minimize the energy variance [50].

For the ground state, the many-body expansion truncated at $M=2$ is exact not only in the noninteracting limit $\gamma=0$ but also in the Tonks-Girardeau limit $\gamma \rightarrow \infty$. In this fermionized limit, the wave function can be written as the modulus of a Vandermonde determinant of plane waves, corresponding to the two-body Jastrow function $u_{2}(r)=\log (\sin r \pi / L)$ [51]. To assess the overall quality of pair wave functions for ground-state properties, we start comparing the variational ground-state energies $E$ obtained for $M=2$ with the exact BA result. In Fig. 1(a), we show the relative error $\Delta E / E$ as a function of the interaction strength $\gamma$. We find that the relative error is lower than $10^{-4}$ for all values of $\gamma$ and the accuracy of our two-body Jastrow function is superior to previously published variational results based on either cMPS [23] or VMC [52] methods [see Fig. 1(a) for a quantitative comparison]. Notice that the improvement with respect to previous VMC results is due to the larger variational freedom of our $u_{2}(r)$ function, which is not restricted to any specific functional form as done in Ref. [52].

Even though the accuracy reached by the two-body Jastrow function may already be sufficient for most practical purposes for all values of $\gamma$, we also consider higher-order terms with $M=3$; see Fig. (2)(a). The introduction of the third-order term yields a sizable improvement such that the maximum error is about 3 orders of magnitude smaller than original cMPS results [23], and features a similar accuracy of recently reported cMPS results [24]. Overall, our approach reaches a precision on a continuous-space system, which is comparable to state-of-the-art MPS or DMRG results for gapless systems on a lattice [53].

Finally, to further assess the quality of our ground-state ansatz beyond the total energy, we also study nonlocal properties of the ground-state wave function, which are not accessible by existing exact BA methods. In Figs. 1(b) and 1(c), we show, respectively, our results for the off-diagonal part of the one-body density matrix, $g_{1}(r) \propto\left\langle\Psi^{\dagger}(r) \Psi(0)\right\rangle$, and for the pair correlation function, $g_{2}(r) \propto\left\langle\Psi^{\dagger}(r) \Psi(r) \Psi^{\dagger}(0) \Psi(0)\right\rangle$, where $\Psi(r)$ is the bosonic

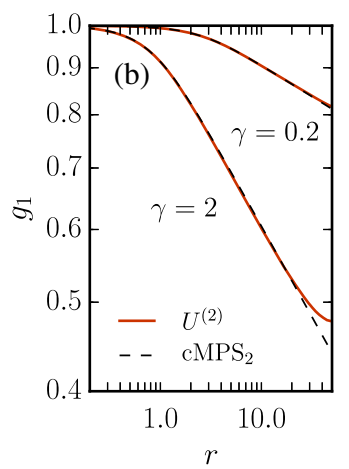

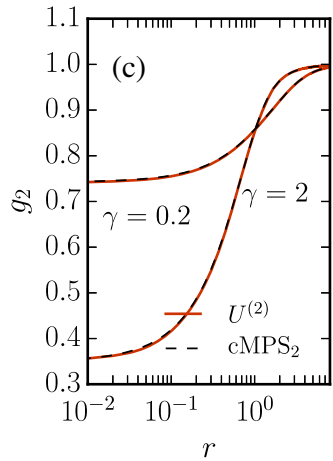

FIG. 1. Ground-state properties of the Lieb-Liniger model as obtained from different variational approaches: (a) relative accuracy of the ground-state energy, (b) one-body density matrix, (c) density-density pair correlation functions. $U^{(2)}$ and $U^{(3)}$ denote results for the two- and three-body expansion (present work), $U_{\mathrm{AG}}^{(2)}$ is the parametrized two-body Jastrow state of Ref. [52], cMPS 1 results are from Ref. [23], and $\mathrm{cMPS}_{2}$ are those very recently reported in Ref. [24]. Distances $r$ are in units of the inverse density $1 / n$. Our variational results are obtained for $N=100$ particles. Finite-size corrections on local quantities are negligible, and very mildly affect the reported large-distance correlations. Overall statistical errors are of the order of symbol sizes for (a) and line widths for (b) and (c). 
field operator. We find an overall excellent agreement with the results that have been obtained with cMPS in Ref. [24], except for some small deviations at large values of $r$, which we attribute to residual finite-size effects in our approach. We find that the addition of the three-body terms does not significantly change correlation functions. Already at the two-body level, the present results are statistically indistinguishable from exact results obtained using our implementation [54] of the worm algorithm [55] and for the same system (not shown).

\section{B. Quench dynamics}

Having assessed the quality of the ansatz for local and nonlocal ground-state properties, we now turn to the study of the out-of-equilibrium properties of the Lieb-Liniger model. We focus on the description of the unitary dynamics induced by a global quantum quench of the interaction strength, from an initial value $\gamma_{i}$ to a final value $\gamma_{f}$. Exact BA results are available only in the case of a noninteracting initial state $\left(\gamma_{i}=0\right)$. Even in this case, the dynamical BA equations can be exactly solved only for a modest number of particles with further truncation in the number of energy eigenmodes [56,57], $N \lesssim 10$, since the complexity of the BA solution increases exponentially with the number of particles. Simplifications in the thermodynamic limit are exploited by the quench action [58], and have recently been applied to quantum quenches starting from a noninteracting initial state [57]. In the following, we first compare our tVMC results to these existing results, and then present new results for quenches following a nonvanishing initial interaction strength.

To assess the quality of the time-dependent wave function, we compare our results for the evolution of local density-density correlations $g_{2}(0, t)$ with the truncated BA results obtained in Refs. [56,59] for a small number of particles, $N \simeq 6$. Appendix $\mathrm{E}$ also provides further validation of our method accessing $g_{2}(r, t)$ at nonvanishing distances. The comparison shown in Fig. 2(a) shows an overall good agreement. The tVMC and BA results are indistinguishable for weak interactions $\left(\gamma_{f}=1\right)$ at the scale of the figure; similarly, for this interaction strength, tVMC calculations based on $U^{(2)}$ and $U^{(3)}$ agree with each other up to possible dephasing effects not visible within our noise level. For larger interactions, we notice systematic but small differences between BA and tVMC with $M=2$ or $M=3$ results. These differences amount to a small increase in the amplitude of the oscillations. This effect tends to increase with the interaction strength, being hardly visible for $\gamma=1$ and more pronounced for $\gamma=10$. However, these oscillations result at large times from the discrete mode structure due to the very small number of particles. They vanish in the physical thermodynamic limit. In turn, the comparison at small particle numbers indicates an accuracy better than a few percent for time-averaged quantities in the asymptotic large time limit up to $\gamma=10$, with results at $M=3$ systematically improving on the $M=2$ case. Concerning small and intermediate time scales, we do not observe systematic deviations between the tVMC results and the BA solution. In particular, the relaxation times are remarkably well captured by the tVMC approach. On the basis of this comparison and of the comparison for three particles with exact diagonalization results presented in Appendix E, we conclude that tVMC approach allows accurate quantitative studies of both the relaxation and equilibration dynamics. This careful benchmarking now allows us to confidently apply the tVMC approach regimes that are inaccessible to exact BA, namely large but finite $N$ and long times, as well as the case of nonvanishing initial interactions.

Let us consider relaxation of density correlations for a large number of particles, close to the thermodynamic limit (here, we use $N=100$ ). As we show in Fig. 2(b), we notice

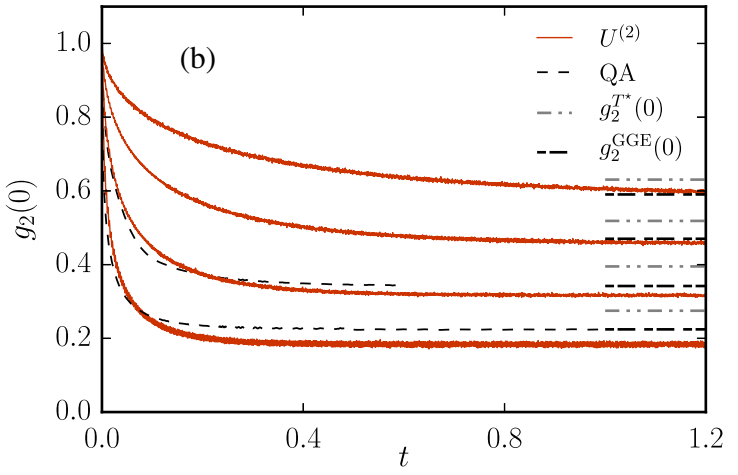

FIG. 2. Time-dependent expectation value of local two-body correlations after a quantum quench from a noninteracting state, $\gamma_{i}=0$, to $\gamma_{f}$. (a) tVMC results are compared with BA results obtained for a small number of particles [56,59]. The correlation function is rescaled to have $g_{2}(0,0)=1$. (b) tVMC results for $N=100$ particles and $\gamma_{f}=1,2,4,8$ (from top to bottom) compared to the quench action (QA) predictions from Ref. [57] (dashed lines), to the Boltzmann thermal averages at the effective temperature $T^{\star}$ (dot-dashed lines), and to the GGE thermal averages prediction (rightmost dashed lines). Statistical error bars on tVMC data are of the order of the line widths. 


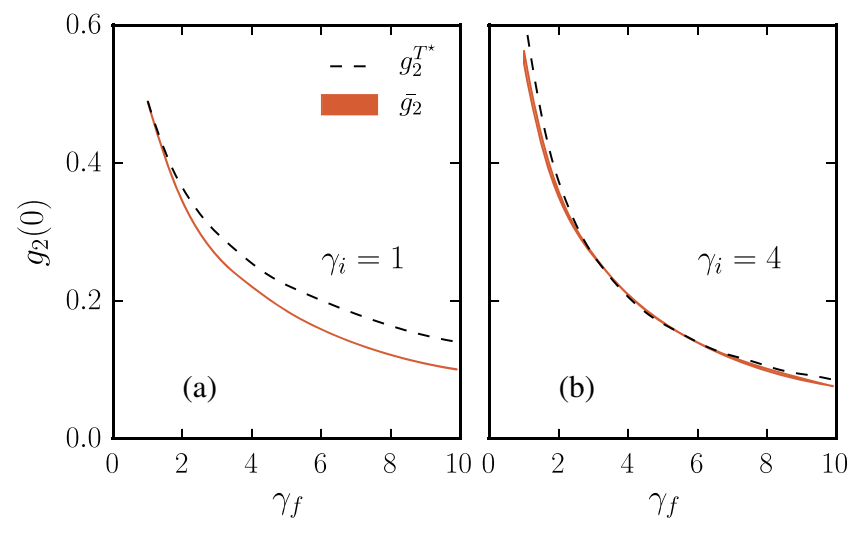

FIG. 3. Time-dependent expectation value of local two-body correlations after a quantum quench from the interacting ground state at $\gamma_{i}=1$ (a) and $\gamma_{i}=4$ (b). Long-term dynamical averages (red continuous lines) are compared to thermal averages at the effective temperature set by energy conservation (black dashed lines). Uncertainties on the thermal averages are of the order of the line width, and are larger for small values of $\gamma_{f}$.

that the amplitudes of the large-time oscillations, attributed to the discrete mode spectrum, are now drastically suppressed compared to the quenches with $N=6$. After an initial relaxation phase, the quantity $g_{2}(0, t)$ approaches a stationary value. Comparing our curves with those obtained with the quench action method, we find a qualitatively good agreement, albeit a general tendency to underestimate the quench action predictions is observed.

We now turn to quenches from interacting initial states $\left(\gamma_{i} \neq 0\right)$ to different interacting final states for which no results have been obtained by means of exact BA nor the simplified quench action method so far. In Fig. 3, we show the asymptotic equilibrium values obtained with our tVMC approach for quantum quenches from $\gamma_{i}=1$ (left-hand panel) and $\gamma_{i}=4$ (right-hand panel) to several values of $\gamma_{f}$. Since, by the variational theorem, the ground state of $\mathcal{H}_{i}$ gives an upper bound for the ground-state energy of $\mathcal{H}_{f}$, the system is pushed into a linear combination of excited states of the final Hamiltonian. For systems able to thermalize to the Boltzmann ensemble (BE), relaxation to a stationary state described by the density matrix $\rho_{T^{\star}}=e^{-\mathcal{H}_{f} / T^{\star}}$, at an effective temperature $T^{\star}$, would occur. Comparing the stationary value $\overline{g_{2}}(0)$ of our tVMC calculations at long times to the thermal values of the pair correlation functions $g_{2}^{T^{\star}}(0)$, a necessary condition for simple Boltzmann thermalization is given by $\overline{g_{2}}=g_{2}^{T^{\star}}$. The effective temperature $T^{\star}$ is determined by imposing the energy expectation value of the final Hamiltonian $\mathcal{H}_{f}$ in the ground state $\Phi_{0}\left(\gamma_{i}\right)$ of the initial Hamiltonian, $\left\langle\mathcal{H}_{f}\right\rangle_{T^{\star}}=\left\langle\Phi_{0}\left(\gamma_{i}\right)\left|\mathcal{H}_{f}\right| \Phi_{0}\left(\gamma_{i}\right)\right\rangle$. Here, the thermal expectation value, $\left\langle\mathcal{H}_{f}\right\rangle_{T^{\star}}$ at the equilibrium temperature $T^{\star}$ is computed from the Yang-Yang BA equations [60]. The quantity $\left\langle\mathcal{H}_{f}\right\rangle_{T^{\star}}$ then depends on a single parameter $T^{\star}$, which is fitted to match the value of $\left\langle\Phi_{0}\left(\gamma_{i}\right)\left|\mathcal{H}_{f}\right| \Phi_{0}\left(\gamma_{i}\right)\right\rangle$.
As we show in Fig. 2(b), Boltzmann thermalization certainly does not occur in the case for the Lieb-Liniger model when quenching from a noninteracting state, $\gamma_{i}=0$, where we find $\overline{g_{2}} \neq g_{2}^{T^{*}}$. This can be understood in terms of the existence of dynamically conserved charges (beyond energy and density conservation), which can yield an equilibrium value substantially different from the $\mathrm{BE}$ prediction. In particular, it is widely believed that the generalized Gibbs ensemble (GGE) is the correct thermal distribution approached after the quench [61,62]. Several constructive approaches for the GGE have been put forward in past years $[8,9,57]$, and the quench action predictions reported in Fig. 2(b) converge to the GGE predictions for the thermal values. In Fig. 2(b), we also show the thermal GGE values $g_{2}^{\mathrm{GGE}}$ (rightmost dashed lines), and note that our results are much closer to the GGE predictions than the simple BE. Deviations from the asymptotic GGE results are observed at large $\gamma_{f}$, a regime in which the accuracy of our approach is still sufficient to resolve the difference between the $\mathrm{BE}$ and the long-term equilibration value.

For correlated initial ground states $\gamma_{i} \neq 0$, GGE predictions are fundamentally harder to obtain than for the noninteracting initial states, and the $\mathrm{BE}$ is the only reference thermal distribution we can compare with at this stage. From our results we observe that the difference between $\overline{g_{2}}$ and the simple BE prediction $g_{2}^{T^{*}}$ is quantitatively reduced; see Fig. 3. In particular, for $\gamma_{i}=4$, the stationary values $\overline{g_{2}}$ are quantitatively close to the ones predicted by the Boltzmann thermal distribution at the effective temperature $T^{\star}$. Even though this quantitative agreement is likely to be coincidental, the regimes of parameter quenches we study here provide guidance for future experimental studies. In particular, it will be of great interest to understand whether a crossover from a strongly non-Boltzmann to a close-to-Boltzmann thermal behavior might occur as a function of the initial interaction strength also for other local observables.

\section{CONCLUSIONS}

In this paper, we introduce a novel approach to the dynamics of strongly correlated quantum systems in continuous space. Our method is based on a correlated many-body wave function systematically expanded in terms of reduced $m$-body Jastrow functions. The unitary dynamics in the subspace of these correlated states is realized using the time-dependent variational Monte Carlo method. We demonstrate the possibility of performing calculations up to the three-body level, $m \leq 3$, for the Lieb-Liniger model, for both static and dynamical properties. The improvement from $m=2$ to $m=3$ provides an internal criterion to judge the validity of our results whenever exact results are unavailable. Benchmarking the tVMC approach with exact or numerical approaches 
whenever available, we find a very good agreement with existing results. For static properties, our approach is at the level of state-of-the-art MPS techniques in lattice systems and of the latest cMPS results for interacting gases. For dynamical properties, we investigate, for the first time, general interaction quenches, which are at the moment unaccessible to Bethe ansatz approaches. Since the general structure of our t-VMC method does not depend on the dimensionality of the system, it can be directly applied to bosonic systems in higher dimensions with a polynomial increase in computational cost. The methods we present here therefore pave the way to accurate out-of-equilibrium dynamics of two- and three-dimensional quantum gases and fluids beyond mean-field approximations.

\section{ACKNOWLEDGMENTS}

We acknowledge discussions with M. Dolfi, J. De Nardis, M. Fagotti, T. Osborne, and M. Troyer. We thank M. Ganahl for providing the cMPS results in Fig. 1, J. Zill for the Bethe ansatz results in Fig. 2(a), and J. De Nardis for the quench actions results in Fig. 2(b). This research was supported by the Marie Curie IEF Program (FP7/20072013 Grant Agreement No. 327143), the European Research Council Starting Grant "ALoGlaDis" (FP7/ 2007-2013 Grant Agreement No. 256294) and Advanced Grant "SIMCOFE" (FP7/2007-2013 Grant Agreement No. 290464), the European Commission FET-Proactive QUIC (H2020 Grant No. 641122), the French ANR-16CE30-0023-03 (THERMOLOC), and the Swiss National Science Foundation through NCCR QSIT. It was performed using HPC resources from GENCI-CCRT/ CINES (Grant No. c2015056853).

\section{APPENDIX A: FUNCTIONAL STRUCTURE OF MANY-BODY TERMS}

The local residuals $R^{(M)}(\mathbf{X} ; t)=i \partial_{t} U^{(M)}(\mathbf{X} ; t)-$ $E_{\mathrm{loc}}^{(M)}(\mathbf{X} ; t)$ are vanishing if the Schrödinger equation is exactly satisfied by the many-body wave function truncated at some order $M$. The local energy $E_{\mathrm{loc}}^{(M)}(\mathbf{X}, t)$, however, may contain effective interaction terms involving a number of bodies larger than $M$, which leads to a systematic error in the truncation. However, the structure of these additional terms stemming from the local energy can be systematically used to deduce the functional structure of the higherorder terms. For example, the one-body truncated local energy reads, for one-dimensional particles,

$$
\begin{aligned}
E_{\mathrm{loc}}^{(1)}(\mathbf{X} ; t)= & -\frac{1}{2} \sum_{i}\left\{\left[\partial_{x_{i}} u_{1}\left(x_{i} ; t\right)\right]^{2}+\partial_{x_{i}}^{2} u_{1}\left(x_{i} ; t\right)\right\} \\
& +\sum_{i} v_{1}\left(x_{i}\right)+\frac{1}{2} \sum_{i \neq j}^{N} v_{2}\left(x_{i}, x_{j}\right),
\end{aligned}
$$

and contains a two-body term that cannot be accounted for exactly by $u_{1}$. Introduction of a symmetric two-body Jastrow factor $u_{2}\left(x_{i}, x_{j} ; t\right)$ then leads to

$$
\begin{aligned}
E_{\mathrm{loc}}^{(2)}(\mathbf{X} ; t)= & E_{\mathrm{loc}}^{(1)}(\mathbf{X} ; t)+ \\
& -\frac{1}{2} \sum_{i \neq j}\left[\partial_{x_{i}} u_{1}\left(x_{i} ; t\right) \partial_{x_{i}} u_{2}\left(x_{i}, x_{j} ; t\right)\right]+ \\
& -\frac{1}{2} \sum_{i \neq j} \partial_{x_{i}}^{2} u_{2}\left(x_{i}, x_{j} ; t\right)+ \\
& -\frac{1}{2} \sum_{i \neq j} \sum_{k \neq i} \partial_{x_{i}} u_{2}\left(x_{i}, x_{j} ; t\right) \partial_{x_{i}} u_{2}\left(x_{i}, x_{k} ; t\right) .
\end{aligned}
$$

In the latter expression, one recognizes an effective twobody term which can be accounted for by $u_{2}$ and an additional three-body term in the form of a product of twobody functions. The functional form of the three-body Jastrow term can therefore be deduced from this additional term and formed accordingly:

$$
u_{3}\left(x_{i}, x_{j}, x_{k} ; t\right)=\bar{u}_{3}\left(x_{i}, x_{j} ; t\right) \bar{u}_{3}\left(x_{j}, x_{k} ; t\right),
$$

with two-body functions $\bar{u}_{3}\left(x_{i}, x_{j} ; t\right)$ containing new variational parameters to be determined. Upon pursuing this approach, the expansion can be systematically pushed to higher orders and the functional structure of the higherorder functions inferred. The same constructive approach we discuss here is also valid for the Schrödinger equation in imaginary time, $\partial_{\tau} U(\mathbf{X} ; \tau)=-E_{\text {loc }}(\mathbf{x} ; \tau)$, and has been successfully used to infer the functional structure for ground-state properties [63].

\section{APPENDIX B: MONTE CARLO SAMPLING}

In order to solve the tVMC equations of motion, Eq. (6), expectation values of some given operator $\mathcal{O}$ need to be computed over the many-body wave function $\Phi(\mathbf{X}, t)$. This is achieved by means of Monte Carlo sampling of the probability distribution $\Pi(\mathbf{X})=|\Phi(\mathbf{X})|^{2}$ (in the following, we omit explicit reference to the time $t$, assuming that all expectation values are taken over the wave function at a given fixed time). An efficient way of sampling the given probability distribution is to devise a Markov chain of configurations $\mathbf{X}(1), \mathbf{X}(2), \ldots, \mathbf{X}\left(N_{c}-1\right), \mathbf{X}\left(N_{c}\right)$, which are distribute according to $\Pi(\mathbf{X})$. Quantum expectation values of a given operator $\mathcal{O}$ can then be obtained as statistical expectation values over the Markov chain as

$$
\frac{\langle\Phi|\mathcal{O}| \Phi\rangle}{\langle\Phi \mid \Phi\rangle} \simeq \frac{1}{N_{c}} \sum_{i=1}^{N_{c}} \mathcal{O}_{\mathrm{loc}}(\mathbf{X}(i)),
$$


where $\mathcal{O}_{\text {loc }}(\mathbf{X})=(\langle\mathbf{X}|\mathcal{O}| \Phi\rangle /\langle\mathbf{X} \mid \Phi\rangle)$, and the equivalence is achieved in the limit $N_{c} \rightarrow \infty$.

The Markov chain is realized by the Metropolis-Hastings algorithm. Given the current state of the Markov chain $\mathbf{X}(i)$, a configuration $\mathbf{X}^{\prime}$ is generated according to a given transition probability $T\left(\mathbf{X}(i) \rightarrow \mathbf{X}^{\prime}\right)$. The proposed configuration is then accepted [i.e., $\mathbf{X}(i+1)=\mathbf{X}^{\prime}$ ] with probability

$A\left(\mathbf{X}(i) \rightarrow \mathbf{X}^{\prime}\right)=\min \left[1, \frac{\Pi\left(\mathbf{X}^{\prime}\right)}{\Pi(\mathbf{X}(i))} \frac{T\left(\mathbf{X}^{\prime} \rightarrow \mathbf{X}(i)\right)}{T\left(\mathbf{X}(i) \rightarrow \mathbf{X}^{\prime}\right)}\right]$

otherwise, it is rejected and $\mathbf{X}(i+1)=\mathbf{X}(i)$.

In the present tVMC calculations, we use simple transition probabilities in which a single particle is displaced, while leaving all the other particle positions unchanged. In particular, a particle index $p$ is chosen with uniform probability $1 / N$ and the position of particle $p$ is then displaced according to $x_{p}^{\prime}=x_{p}+\eta_{\Delta}$, where $\eta_{\Delta}$ is a random number uniformly distributed in $[-(\Delta / 2),(\Delta / 2)]$. The amplitude $\Delta$ is an adjustable parameter, and it can typically be chosen to be of the order of the average interparticle distance. With this choice, the transition probability is simply

$$
T\left(x_{p} \rightarrow x_{p}^{\prime}\right)=\frac{1}{N \Delta},
$$

and the acceptance probability is therefore given by the mere ratio of the probability distributions, $\Pi\left(\mathbf{X}^{\prime}\right) / \Pi(\mathbf{X}(i))$.

\section{APPENDIX C: FINITE-BASIS PROJECTION}

The numerical solution of the equations of motion [Eq. (6)] requires the projection of the Jastrow fields $u_{m}(\mathbf{r} ; t)$ onto a finite basis. The continuous variable $\mathbf{r}$ is reduced to a finite set of $P$ values for each order $m$, $(m, \mathbf{r}) \rightarrow\left(r_{1, m}, r_{2, m}, \ldots, r_{P, m}\right)$. We introduce a super-index $K$ spanning all possible values of the discrete variables $r_{i, m}$. The complete set of variational parameters resulting from the projection on the finite basis can then be written as $u_{K}(t)$ and the associated functional derivatives read $\rho_{K}(t)$.

The integro-differential equations [Eq. (6)] are then brought to the algebraic form,

$$
\sum_{K^{\prime}} S_{K, K^{\prime}} \dot{u}_{K^{\prime}}(t)=-i\left\langle E_{\mathrm{loc}}(t) \rho_{K}(t)\right\rangle,
$$

where we introduce the Hermitian correlation matrix:

$$
\begin{aligned}
S_{K, K^{\prime}}(t) & =\frac{\partial\left\langle\rho_{K}(t)\right\rangle_{t}}{\partial u_{K^{\prime}}} \\
& =\left\langle\rho_{K}(t) \rho_{K^{\prime}}(t)\right\rangle_{t}-\left\langle\rho_{K}(t)\right\rangle_{t}\left\langle\rho_{K^{\prime}}(t)\right\rangle_{t} .
\end{aligned}
$$

At a given time, all the expectations values in Eq. (C1) can be explicitly computed with the stochastic approach described in Appendix B. We are therefore left with a linear system in the $n_{\text {var }}$ unknowns $\dot{u}_{K}(t)$, which needs to be solved at each time $t$.

In the presence of a large number of variational parameters $n_{\text {var }}$, the solution of the linear system can be achieved using iterative solvers, e.g., conjugate gradient methods, which do not need to explicitly form the matrix $S$. Calling $n_{\text {iter }}$ the number of iterations needed to obtain a solution for the linear system, the computational cost to solve Eq. (C1) is $\mathcal{O}(M \times$ $\left.n_{\text {var }} \times n_{\text {iter }}\right)$ as opposed to the $\mathcal{O}\left(M \times n_{\text {var }}^{2}\right)$ operations needed by a standard solver in which the matrix $S$ is formed explicitly. In the present work, we resort to the minimal residual method, which is a variant of the Lanczos method, working in the Krylov subspace spanned by the repeated action of the matrix $S$ onto an initial vector. In typical applications we obtain that $n_{\text {iter }} \ll n_{\text {var }}$, and several thousands of variational parameters can be efficiently treated. This is of fundamental importance when the continuous (infinite-basis) limit must be taken, for which $n_{\mathrm{var}} \rightarrow \infty$.

Once the unknowns $\dot{u}_{K}(t)$ are determined, we can solve numerically the first-order differential equations given in Eq. (6) for given initial conditions $u_{K}(0)$. In the present work, we adopt an adaptive fourth-order Runge-Kutta scheme for the integration of the differential equations.

\section{APPENDIX D: LATTICE REGULARIZATION FOR THE LIEB-LINIGER MODEL}

We consider a general wave function $\Psi\left(x_{1}, x_{2}, \ldots, x_{N}\right)$ for $N$ one-dimensional particles, governed by the LiebLiniger Hamiltonian. By means of the variational Monte Carlo method, we want to sample $|\Phi(\mathbf{X})|^{2}$. This is achieved via a lattice regularization, i.e.,

$$
\int d \mathbf{X}|\Phi(\mathbf{X})|^{2} \simeq \sum_{l_{1}, l_{2} \ldots l_{N}}\left|\Phi\left(l_{1}, l_{2}, \ldots, l_{N}\right)\right|^{2},
$$

where $l_{i}=\{0, a, \ldots, L-a\}$ are discrete particle positions, $a$ the lattice spacing, $L$ the box size and $N_{s}=1+L / a$ the number of lattice sites. As a discretized Hamiltonian, we take

$$
\begin{aligned}
H_{a} \Phi\left(l_{1} \ldots l_{N}\right)= & -\frac{\hbar^{2}}{2 m a^{2}} \sum_{i}\left\{\frac{4}{3}\left[\Phi\left(l_{1} \ldots l_{i}-a, \ldots, l_{N}\right)+\Phi\left(l_{1} \ldots l_{i}+a, \ldots, l_{N}\right)\right]\right. \\
& \left.-\frac{1}{12}\left[\Phi\left(l_{1} \ldots l_{i}-2 a, \ldots, l_{N}\right)+\Phi\left(l_{1} \ldots l_{i}+2 a, \ldots, l_{N}\right)\right]+-\frac{5}{2} \Phi\left(l_{1} \ldots l_{i}, \ldots, l_{N}\right)\right\}+\Phi\left(l_{1} \ldots l_{N}\right) \frac{g}{a} \sum_{i<j} \delta\left(l_{i}, l_{j}\right) .
\end{aligned}
$$


The first terms constitute just the fourth-order approximation of the Laplacian via central finite differences, whereas the last term corresponds to the two-body delta interaction part.

With this discretization, a two-body Jastrow factor reads

$$
u_{2}\left(x_{i j} ; t\right)=u_{2}\left(l_{i}, l_{j} ; t\right)
$$

where $u_{2}(a, b ; t)$ is a time-dependent matrix of size $N_{s} \times N_{s}$, which, in 1D and in the presence of translational symmetry, depends only on $\operatorname{dist}(a-b)$; i.e., it has $N_{s} / 2$ variational parameters.

\section{APPENDIX E: BENCHMARK STUDY FOR $N=3$ ON A LATTICE}

Here, we use exact diagonalization of a Hamiltonian within a given finite basis for a quantitative test of our method. Exact diagonalization is limited to very small systems on a finite basis, and we choose a system containing $N=3$ particles on $L / a=40$ lattice sites as a simple, but highly nontrivial reference. In contrast to our comparison with BA methods, all observables can be accessed by exact diagonalization, and we use the off-diagonal one-body density matrix $g_{1}(r, t)$ and the pair correlation function $g_{2}(r, t)$ at different distances $r=\left|x_{1}-x_{2}\right|$ of two particles after time $t$, where the system is quenched from the noninteracting initial state, $\gamma_{i}=0$, to a final interaction, $\gamma_{f}>0$, to provide a benchmark on a more general observable.

We first benchmark the influence of the time-step lattice size discretization $\Delta t$ error on $g_{2}$. From Fig. 4(a), we see that the tVMC dynamics is stable over a long time and the time step error can be brought to convergence. Further, we see that for final interaction $\gamma_{f}=4$, the truncation at the two-body level $U^{(2)}$ introduces only a small systematic error, mainly a dephasing effect, which is almost negligible at the scale of the figure. Because of the stochastic noise of the Monte Carlo integration, tVMC introduces additional high-frequency oscillations which are, however, well

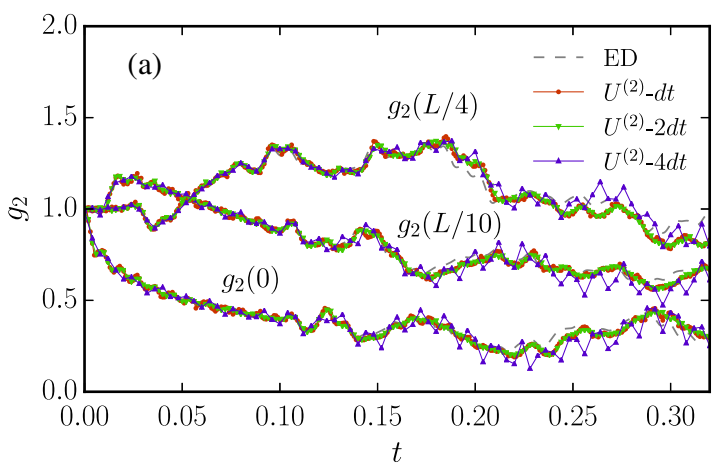

separable from the deterministic propagation. The amplitude of these high-frequency oscillations also quantifies the purely statistical error of our data.

Whereas exact diagonalization is limited to rather small basis sets, we can access a much larger basis within tVMC method. In Fig. 4(b), we show results within the $U^{(2)}$ approximation with $L / a=80$ and $L / a=160$ with time discretization $\Delta t \sim(L / a)^{2}$. We see that the basis set truncation in general introduces a dephasing at large enough time.

The systematic error of $U^{(2)}$ increases towards quenches to stronger interaction strength and becomes more visible for $\gamma_{f}=8$; see Fig. 5(a). However, even in this case, the most important effect remains to be a simple dephasing; a small shift of averaged quantities is probable, but difficult to quantify precisely. Introducing general three-body Jastrow fields $U^{(3)}$, described in detail in Appendix F, the systematic error for $N=3$ can be fully eliminated.

In Fig. 5(b), we also benchmark the possibility of calculating the off-diagonal one-body density matrix after a quench. Here, the systematic error of $U^{(2)}$ is more pronounced at smaller $\gamma_{f}$ in the long range and long time regime.

From our study of the three-particle problem, we conclude that truncation of the many-body wave function at the level of $U_{2}$ may provide an excellent approximation for $g_{2}(r, t)$ for quenches involving not too strong interaction strengths, $\gamma \lesssim 5$. The systematic error due to the $U^{(2)}$ truncation is mainly a dephasing at large times involving small relative errors of time-averaged quantities. Similar systematic dephasing errors will occur for too large time discretization or basis set truncation.

Since our method provides a parametrization of the full wave function for a given time, many different observables can be evaluated via usual Monte Carlo methods. However, the quality of different observables may vary and depend more sensitively on the inclusion of higher-order

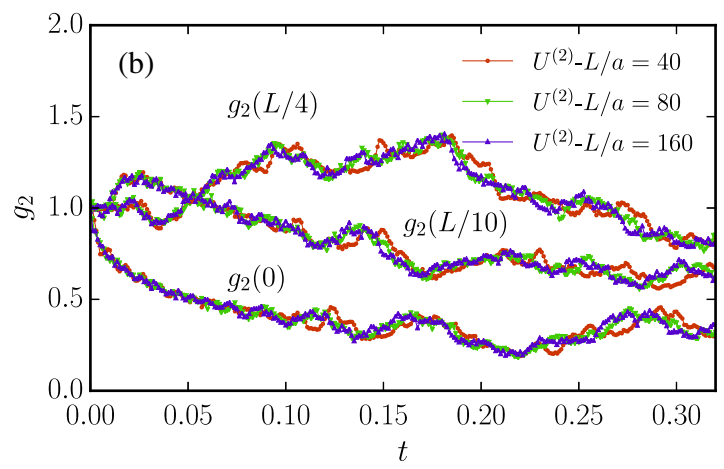

FIG. 4. Time-dependent expectation value of the two-body correlations after a quantum quench from a noninteracting state, $\gamma_{i}=0$, to $\gamma_{f}=4$ at three different distances, $\left|x_{1}-x_{2}\right|=0, L / 10, L / 4$. Here, the system is on a lattice with $L / a=40$ lattice sites. The full line is obtained by exact diagonalization (ED) of the Hamiltonian; the other curves are from tVMC results truncated at the level of $U^{(2)}$. In (a), we show the convergence with different time-step discretization. In (b), we show the approach to the continuum for tVMC simulations using $U^{(2)}$ for discretizations $L / a=40,80$, and 160 . 

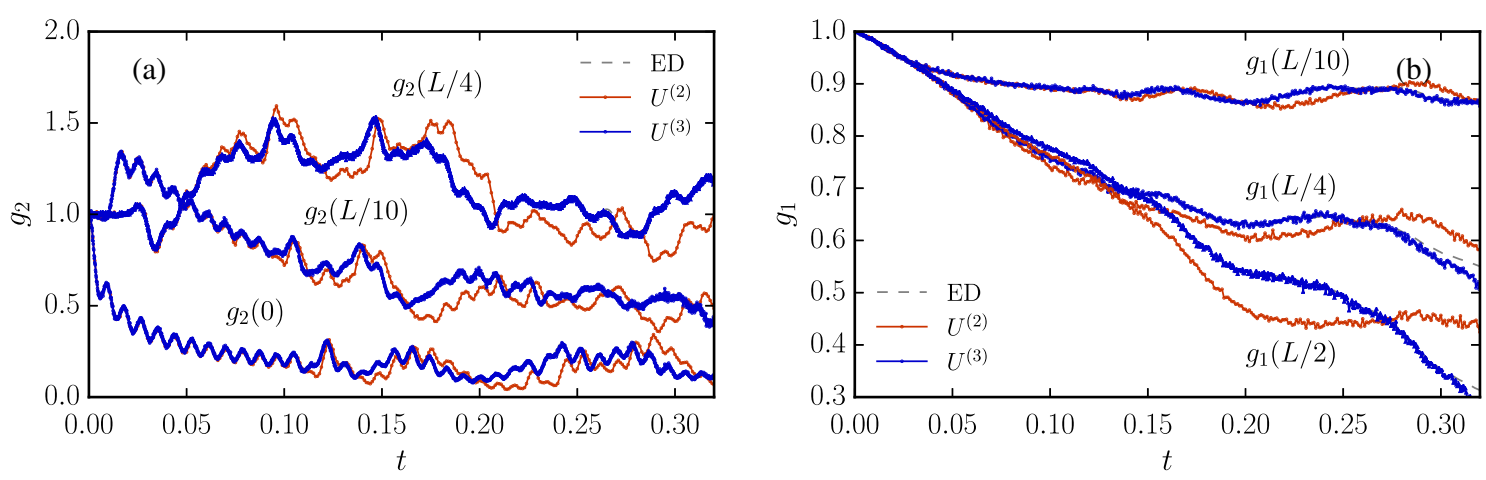

FIG. 5. (a) Time-dependent expectation value of the two-body correlations $g_{2}(r, t)$ after a quantum quench from a noninteracting state, $\gamma_{i}=0$, to $\gamma_{f}=8$ at three different distances, $\left|x_{1}-x_{2}\right|=0, L / 10, L / 4$. Here, the system is on a lattice with $L / a=40$ lattice sites. The full line is obtained by exact diagonalization of the Hamiltonian; the other curves are from tVMC results truncated at the level of $U^{(2)}$ or $U^{(3)}$. In contrast to $\gamma_{f}=4$ shown in Fig. 4 , systematic differences of $U^{(2)}$ compared to the exact results are more visible here; the exact dynamics is recovered by inclusion of three-body terms $U^{(3)}$ into the tVMC wave function. (b) Off-diagonal single-particle density matrix $g_{1}(r, t)$ at three different distances, $r=L / 10, L / 4$, and $L / 2$. The exact results (ED-black dashed line) are for all purposes indistinguishable from the $U^{(3)}$ curves.

correlations $U^{(n)}$ with $n>2$, as in the case of the singlebody density matrix. Although these higher-order terms are computationally expensive, the scaling is not exponential, and we explicitly show that calculations with $n=3$ are feasible. We note that the computational complexity may be further reduced by functional forms adapted to the problem [26].

\section{APPENDIX F: GENERAL STRUCTURE OF $U^{(3)}$}

For a general time-dependent wave function, we have to go beyond the usual ground-state structure of the threebody Jastrow term given in Eq. (A3). Here, we provide details of our three-body term in a general form beyond the present application in one dimension.

Introducing $M$ basis functions, $b^{a}(r), a=1, \ldots, M$, we can introduce many-body vectors [26], $B_{i \alpha}^{a}=\sum_{j} \mathbf{r}_{i j}^{\alpha} b^{a}\left(r_{i j}\right)$, where $\alpha=1, \ldots, D$ indicates the summation over directions and $i=1, \ldots, N$. The variational parameters of a general three-body structure can then be written in terms of a matrix $w_{a b}$, such that

$$
\sum_{i \neq j \neq k} u_{3}\left(\mathbf{r}_{1}, \mathbf{r}_{2}, \mathbf{r}_{3}\right)=\sum_{a b} w_{a b} W^{a b}, \quad W^{a b}=\sum_{i \alpha} B_{i \alpha}^{a} B_{i \alpha}^{b}
$$

In order to reduce the variational parameters $\left(\sim M^{2}\right)$, we may perform a singular value decomposition of the matrix $w_{a b}$ to reduce the effective degrees of freedom.

[1] Nature Physics Insight on Non-equilibrium physics, Nat. Phys. 11, 103 (2015).
[2] D. Ceperley, Path Integrals in the Theory of Condensed Helium, Rev. Mod. Phys. 67, 279 (1995).

[3] L. Pollet, Recent Developments in Quantum Monte Carlo Simulations with Applications for Cold Gases, Rep. Prog. Phys. 75, 094501 (2012).

[4] T. Plisson, B. Allard, M. Holzmann, G. Salomon, A. Aspect, P. Bouyer, and T. Bourdel, Coherence Properties of a TwoDimensional Trapped Bose Gas around the Superfluid Transition, Phys. Rev. A 84, 061606 (2011).

[5] N. G. Berloff and B. V. Svistunov, Scenario of Strongly Nonequilibrated Bose-Einstein Condensation, Phys. Rev. A 66, 013603 (2002).

[6] N. Navon, A. L. Gaunt, R. P. Smith, and Z. Hadzibabic, Emergence of a Turbulent Cascade in a Quantum Gas, Nature (London) 539, 72 (2016).

[7] M. Gaudin, La Fonction d'Onde de Bethe (Masson, Paris, 1983).

[8] J.-S. Caux and R. M. Konik, Constructing the Generalized Gibbs Ensemble after a Quantum Quench, Phys. Rev. Lett. 109, 175301 (2012).

[9] J.-S. Caux and F. H. L. Essler, Time Evolution of Local Observables After Quenching to an Integrable Model, Phys. Rev. Lett. 110, 257203 (2013).

[10] M. Collura, S. Sotiriadis, and P. Calabrese, Equilibration of a Tonks-Girardeau Gas Following a Trap Release, Phys. Rev. Lett. 110, 245301 (2013).

[11] S. Trotzky, Y-A. Chen, A. Flesch, I. P. McCulloch, U. Schollwöck, J. Eisert, and I. Bloch, Probing the Relaxation Towards Equilibrium in an Isolated Strongly Correlated One-Dimensional Bose Gas, Nat. Phys. 8, 325 (2012).

[12] T. Langen, R. Geiger, M. Kuhnert, B. Rauer, and J. Schmiedmayer, Local Emergence of Thermal Correlations in an Isolated Quantum Many-Body System, Nat. Phys. 9, 640 (2013).

[13] D. Greif, G. Jotzu, M. Messer, R. Desbuquois, and T. Esslinger, Formation and Dynamics of Antiferromagnetic Correlations in Tunable Optical Lattices, Phys. Rev. Lett. 115, 260401 (2015). 
[14] M. Gring, M. Kuhnert, T. Langen, T. Kitagawa, B. Rauer, M. Schreitl, I. Mazets, D. A. Smith, E. Demler, and J. Schmiedmayer, Relaxation and Prethermalization in an Isolated Quantum System, Science 337, 1318 (2012).

[15] M. Cominotti, D. Rossini, M. Rizzi, F. Hekking, and A. Minguzzi, Optimal Persistent Currents for Interacting Bosons on a Ring with a Gauge Field, Phys. Rev. Lett. 113, 025301 (2014).

[16] P. Calabrese and J. Cardy, Time Dependence of Correlation Functions Following a Quantum Quench, Phys. Rev. Lett. 96, 136801 (2006).

[17] S. R. White, Density Matrix Formulation for Quantum Renormalization Groups, Phys. Rev. Lett. 69, 2863 (1992).

[18] S. R. White and A. E. Feiguin, Real-Time Evolution Using the Density Matrix Renormalization Group, Phys. Rev. Lett. 93, 076401 (2004).

[19] A. J. Daley, C. Kollath, U. Schollwock, and G. Vidal, TimeDependent Density-Matrix Renormalization-Group Using Adaptive Effective Hilbert Spaces, J. Stat. Mech. (2004) P04005.

[20] F. B. Anders and A. Schiller, Real-Time Dynamics in Quantum-Impurity Systems: A Time-Dependent Numerical Renormalization-Group Approach, Phys. Rev. Lett. 95, 196801 (2005).

[21] M. Dolfi, B. Bauer, M. Troyer, and Z. Ristivojevic, Multigrid Algorithms for Tensor Network States, Phys. Rev. Lett. 109, 020604 (2012).

[22] M. Dolfi, A. Kantian, B. Bauer, and M. Troyer, Minimizing Nonadiabaticities in Optical-Lattice Loading, Phys. Rev. A 91, 033407 (2015).

[23] F. Verstraete and J. I. Cirac, Continuous Matrix Product States for Quantum Fields, Phys. Rev. Lett. 104, 190405 (2010).

[24] M. Ganahl, J. Rincon, and G. Vidal, Continuous Matrix Product States for Quantum Fields: An Energy Minimization Algorithm., Phys. Rev. Lett. 118, 220402 (2017).

[25] C. J. Umrigar, J. Toulouse, C. Filippi, S. Sorella, and R. G. Hennig, Alleviation of the Fermion-Sign Problem by Optimization of Many-Body Wave Functions, Phys. Rev. Lett. 98, 110201 (2007).

[26] M. Holzmann, B. Bernu, and D. M. Ceperley, Many-Body Wavefunctions for Normal Liquid ${ }^{3} \mathrm{He}$, Phys. Rev. B 74, 104510 (2006).

[27] M. Taddei, M. Ruggeri, S. Moroni, and M. Holzmann, Iterative Backflow Renormalization Procedure for ManyBody Ground-State Wave Functions of Strongly Interacting Normal Fermi Liquids, Phys. Rev. B 91, 115106 (2015).

[28] G. Carleo, F. Becca, M. Schiro, and M. Fabrizio, Localization and Glassy Dynamics Of Many-Body Quantum Systems., Sci. Rep. 2, 243 (2012).

[29] G. Carleo, F. Becca, L. Sanchez-Palencia, S. Sorella, and M. Fabrizio, Light-Cone Effect and Supersonic Correlations in One- and Two-Dimensional Bosonic Superfluids, Phys. Rev. A 89, 031602 (2014).

[30] L. Cevolani, G. Carleo, and L. Sanchez-Palencia, Protected Quasilocality in Quantum Systems with Long-Range Interactions, Phys. Rev. A 92, 041603 (2015).

[31] B. Blaß and H. Rieger, Test of Quantum Thermalization in the Two-Dimensional Transverse-Field Ising Model, Sci. Rep. 6, 38185 (2016).
[32] G. Carleo and M. Troyer, Solving the Quantum Many-Body Problem with Artificial Neural Networks, Science 355, 602 (2017).

[33] K. Ido, T. Ohgoe, and M. Imada, Time-Dependent ManyVariable Variational Monte Carlo Method for Nonequilibrium Strongly Correlated Electron Systems, Phys. Rev. B 92, 245106 (2015).

[34] We have conveniently set the particle mass and the reduced Planck constant to unity.

[35] A. Bijl, The Lowest Wave Function of the Symmetrical Many Particles System, Physica (Amsterdam) 7, 869 (1940).

[36] R. B. Dingle, The Zero-Point Energy of a System of Particles, Philos. Mag. 40, 573 (1949).

[37] R. Jastrow, Many-Body Problem with Strong Forces, Phys. Rev. 98, 1479 (1955).

[38] E. Feenberg, Theory of Quantum Fluids, Pure and Applied Physics (Academic Press, New York, 1969).

[39] C. L. Kane, S. Kivelson, D. H. Lee, and S. C. Zhang, General Validity of Jastrow-Laughlin Wave Functions, Phys. Rev. B 43, 3255 (1991).

[40] P. A. M. Dirac, Note on Exchange Phenomena in the Thomas Atom, Math. Proc. Cambridge Philos. Soc. 26, 376 (1930).

[41] I. Frenkel, Wave Mechanics: Advanced General Theory, in The International Series of Monographs on Nuclear Energy: Reactor Design Physics Vol. 2 (Clarendon Press, Oxford, 1934).

[42] The natural norm induced by a quantum Hilbert space is the Fubini-Study norm, which is gauge invariant and therefore insensitive to the unknown normalizations of the quantum states we are dealing with here.

[43] E. H. Lieb and W. Liniger, Exact Analysis of an Interacting Bose Gas. I. The General Solution and the Ground State, Phys. Rev. 130, 1605 (1963).

[44] H. Moritz, T. Stoferle, M. Kohl, and T. Esslinger, Exciting Collective Oscillations in a Trapped 1D Gas, Phys. Rev. Lett. 91, 250402 (2003).

[45] T. Kinoshita, T. Wenger, and D.S. Weiss, A Quantum Newton's Cradle, Nature (London) 440, 900 (2006).

[46] J. P. Ronzheimer, M. Schreiber, S. Braun, S. S. Hodgman, S. Langer, I. P. McCulloch, F. Heidrich-Meisner, I. Bloch, and U. Schneider, Expansion Dynamics of Interacting Bosons in Homogeneous Lattices in One and Two Dimensions, Phys. Rev. Lett. 110, 205301 (2013).

[47] B. Fang, G. Carleo, A. Johnson, and I. Bouchoule, QuenchInduced Breathing Mode of One-Dimensional Bose Gases, Phys. Rev. Lett. 113, 035301 (2014).

[48] G. Boéris et al., Mott Transition for Strongly Interacting One-Dimensional Bosons in a Shallow Periodic Potential, Phys. Rev. A 93, 011601 (2016).

[49] S. Sorella, Generalized Lanczos Algorithm for Variational Quantum Monte Carlo, Phys. Rev. B 64, 024512 (2001).

[50] C. J. Umrigar and C. Filippi, Energy and Variance Optimization of Many-Body Wave Functions, Phys. Rev. Lett. 94, 150201 (2005).

[51] M. Girardeau, Relationship between Systems of Impenetrable Bosons and Fermions in One Dimension, J. Math. Phys. (N.Y.) 1, 516 (1960).

[52] G. E. Astrakharchik and S. Giorgini, Correlation Functions and Momentum Distribution of One-Dimensional Bose Systems, Phys. Rev. A 68, 031602 (2003). 
[53] P. Pippan, S. R. White, and H. G. Evertz, Efficient MatrixProduct State Method for Periodic Boundary Conditions, Phys. Rev. B 81, 081103 (2010).

[54] G. Carleo, G. Boéris, M. Holzmann, and L. SanchezPalencia, Universal Superfluid Transition and Transport Properties of Two-Dimensional Dirty Bosons, Phys. Rev. Lett. 111, 050406 (2013).

[55] M. Boninsegni, N. Prokof'ev, and B. Svistunov, Worm Algorithm for Continuous-Space Path Integral Monte Carlo Simulations, Phys. Rev. Lett. 96, 070601 (2006).

[56] J. C. Zill, T. M. Wright, K. V. Kheruntsyan, T. Gasenzer, and M. J. Davis, Relaxation Dynamics of the Lieb-Liniger Gas Following an Interaction Quench: A Coordinate Bethe-Ansatz Analysis, Phys. Rev. A 91, 023611 (2015).

[57] J. De Nardis, L. Piroli, and J.-S. Caux, Relaxation Dynamics of Local Observables in Integrable Systems., J. Phys. A 48, 43FT01 (2015).

[58] J.-S. Caux and F. H. L. Essler, Time Evolution of Local Observables After Quenching to an Integrable Model, Phys. Rev. Lett. 110, 257203 (2013).
[59] J. C. Zill, T. M. Wright, K. V. Kheruntsyan, T. Gasenzer, and M. J. Davis, A Coordinate Bethe Ansatz Approach to the Calculation of Equilibrium and Nonequilibrium Correlations of the One-Dimensional Bose Gas, New J. Phys. 18, 045010 (2016).

[60] C. N. Yang and C.P. Yang, Thermodynamics of a One-Dimensional System of Bosons with Repulsive DeltaFunction Interaction, J. Math. Phys. (N.Y.) 10, 1115 (1969).

[61] M. Rigol, Fundamental Asymmetry in Quenches between Integrable and Nonintegrable Systems, Phys. Rev. Lett. 116, 100601 (2016).

[62] M. Kollar and M. Eckstein, Relaxation of a OneDimensional Mott Insulator after an Interaction Quench, Phys. Rev. A 78, 013626 (2008).

[63] M. Holzmann, D. M. Ceperley, C. Pierleoni, and K. Esler, Backflow Correlations for the Electron Gas and Metallic Hydrogen, Phys. Rev. E 68, 046707 (2003). 